\title{
Die Nationale Kohorte
}

Chronische Krankheiten sind in Deutschland ebenso wie in anderen westlichen Industrieländern die Haupttodesursache. Durch den demografischen Wandel wird die Bedeutung dieser sogenannten Volkskrankheiten in den kommenden Jahrzehnten weiter zunehmen und eine große Belastung für das Gesundheitssystem darstellen. Eine starke Erhöhung der Patientenanzahl wird besonders für Diabetes mellitus, Schlaganfälle, Lungenerkrankungen, Krebs sowie neuropsychiatrische Erkrankungen erwartet [1]. Die Folgen sind steigende Kosten für die $\mathrm{Ku}$ rativmedizin, Rehabilitation und Pflege. Beispielsweise belaufen sich aktuelle Schätzungen für die direkten kurativmedizinischen Kosten von Herz-KreislaufErkrankungen auf $35 \mathrm{Mrd}$. Euro jährlich, für die von Diabetes auf 5,6 Mrd. und für die von Krebs 15 Mrd. Euro [1].

Gesundheitsökonomische Analysen zeigen, dass die Krankheitsvorbeugung (Prävention) ein kostengünstigeres Mittel als die kurative Medizin ist, um die Morbidität und Mortalität bei den meisten chronischen Erkrankungen zu senken $[2,3]$.

Zur Entwicklung effektiver Strategien für die Prävention chronischer Krankheiten besteht weiter großer Forschungsbedarf. Als Grundlage hierfür sind exakte Daten über die Ursachen dieser Erkrankungen und über die Effekte des Vermeidens oder Verringerns ihrer wichtigsten Risikofaktoren erforderlich. Diese Daten werden in epidemiologischen Studien gewonnen; das hierfür optimale Studiendesign ist die prospektive Kohortenstudie. Auf diese Weise können durch wiederholte medi- zinische Untersuchungen, wiederholte Befragungen und Entnahmen von Blutproben Informationen über die Studienteilnehmer vor der eventuellen Diagnose einer Krankheit gesammelt werden. Somit können für eine Vielfalt von $\mathrm{Ge}$ sundheitszuständen oder Krankheitskombinationen (Multimorbidität) die Auswirkungen von Lebensstil, Umwelt und genetischer Konstellationen gemeinsam untersucht und umfassend, valide und verlässlich quantifiziert werden.

Bisher gibt es in Deutschland einige mittelgroße Kohortenstudien mit zusammen etwa 100.000 bis 120.000 Teilnehmern. $\mathrm{Zu}$ nennen sind hier zum Beispiel folgende Studien: CARLA (Cardiovascular Disease, Living and Ageing in Halle), EPIC (European Prospective Investigation into Cancer and Nutrition in Heidelberg und Potsdam), KORA (kooperative Gesundheitsforschung im Raum Augsburg), Heinz Nixdorf Recall Studie (Risk Factors, Evaluation of Coronary Calcification and Lifestyle) in Essen und SHIP (Study of Health In Pomerania in Greifswald) $[4,5,6,7,8]$. Viele wurden jedoch unabhängig voneinander geplant und haben unterschiedliche Studienelemente. Daher können Daten aus den bestehenden Kohortenstudien nur in einem eingeschränkten Umfang zusammengefasst und für gemeinsame wissenschaftliche Auswertungen genutzt werden. Zudem liegt der Median der Altersverteilung bei den Probanden in den meisten Studien mittlerweile bei deutlich über 55 Jahren, sodass die frühe Entwicklung von Krankheiten oft nicht untersucht werden kann. Hinzu kommt, dass viele der existierenden Kohortenstudien zu einer Zeit ini- tiiert wurden (vor bis zu 25 Jahren), als zahlreiche der heute üblichen Untersuchungstechniken noch nicht verfügbar waren. Des Weiteren werden bei einigen der größeren deutschen Kohortenstudien die Bioproben der für die Forschung interessanten Personen in den nächsten zehn bis 20 Jahren größtenteils aufgebraucht sein.

Daher hat ein Netzwerk von Wissenschaftlerinnen und Wissenschaftlern aus der Helmholtz-Gemeinschaft, den Universitäten, der Leibniz-Gemeinschaft und der Ressortforschung deutschlandweit die Initiative zum Aufbau einer groß angelegten, bevölkerungsbezogenen Langzeit-Kohortenstudie ergriffen [9]. Diese Nationale Kohorte wird eine hoch standardisierte und umfassende Datenbasis liefern, die der Heterogenität der deutschen Bevölkerung sowohl in Bezug auf Risikofaktoren als auch auf die bedeutendsten Erkrankungen Rechnung trägt. Die Nationale Kohorte zielt auch darauf ab, ein breites Spektrum von Biomaterialien - auch wiederholt - zu sammeln und für wissenschaftliche Analysen zur Verfügung zu stellen. Auf diese Weise wird die Bearbeitung einer Vielzahl von Forschungsthemen mit ausreichender statistischer Power ermöglicht, was mit bestehenden deutschen Kohorten nicht umsetzbar wäre (zur Berechnung der statistischen Power siehe [13]).

Ähnlich große Kohortenstudien in Europa sind die UK Biobank in Großbritannien, CONSTANCES in Frankreich und LifeGene in Schweden [10, 11, 12]. Gegenüber diesen Studien wird die Nationale Kohorte einige Besonderheiten aufweisen, wie zum Beispiel die Bereit- 


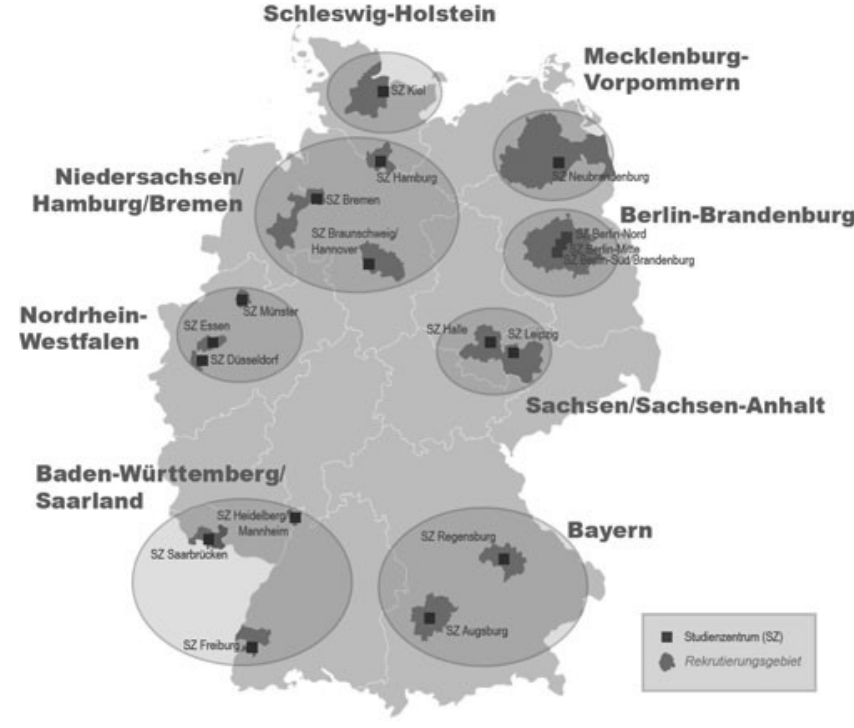

Abb. $1<$ Rekrutierungscluster (Kreise), Studienzentren und Rekrutierungsgebiete der Nationalen Kohorte stellung hochwertiger Bioproben durch eine hochgradig standardisierte Präanalytik und automatische Lagerung, die wiederholte Untersuchung aller Probanden nach fünf Jahren, eine detaillierte Untersuchung präklinischer Krankheitsstadien und die Implementierung der Magnetresonanztomographie (MRT) als zeitgemäße Bildgebungstechnik in der medizinischen Forschung [13].

Das wissenschaftliche Konzept der Nationalen Kohorte wurde durch das Epidemiologische Planungskomitee (EPC) und das Projektmanagement Team - auf der Basis intensiver Vorarbeiten der Thematischen Arbeitsgruppen - ausgearbeitet [14, 15]. Die Thematischen Arbeitsgruppen bestehen nicht nur aus Epidemiologen, sondern aus Wissenschaftlern aus vielen medizinischen Fächern sowie aus den $\mathrm{Na}$ tur- und Geisteswissenschaften. Diese Experten wurden vom EPC ausgewählt, um bei spezifischen Themen, wie zum Beispiel den wichtigsten Krankheitsbildern oder MRT, aus ihrer fachlichen Perspektive heraus Ratschläge zum Einsatz geeigneter Instrumente zu erhalten.

Die Details des Konzepts zur Nationalen Kohorte sollen im Folgenden näher vorgestellt werden.

\section{Wissenschaftliche Fragestellungen und Ziele der Nationalen Kohorte}

Die Nationale Kohorte hat im Wesentlichen vier Hauptziele:

- die Ursachen chronischer Krankheiten und ihren Zusammenhang mit genetischen, Lebensstil- und Umweltfaktoren aufzuklären,

- neue Risikofaktoren zu identifizieren und zur Aufklärung der bestehenden geografischen und sozioökonomischen Ungleichheiten im Gesundheitszustand und Krankheitsrisiko in Deutschland beizutragen,

- Risikovorhersagemodelle für chronische Erkrankungen zu entwickeln und Wege einer wirksamen Vorbeugung aufzuzeigen (personalisierte Präventionsstrategien) sowie

- Möglichkeiten zur Früherkennung chronischer Krankheiten zu identifizieren (Evaluation von Markern als effektive Hilfsmittel zur Krankheitsprävention).

Folgende chronische Krankheiten spielen in der Nationalen Kohorte eine besondere Rolle: kardiovaskuläre Erkrankungen (v. a. Herzinfarkt, Schlaganfall), Diabetes mellitus, Krebs (vor allem Brust-, Darm-, Prostata- und Lungenkrebs), neurodegenerative und neuropsychiatrische Krankheiten (vor allem Demenz, Depression), Erkrankungen der Atemwege (vor allem chronisch obstruktive Lungenerkrankung
(COPD), Asthma) und Infektionskrankheiten (vor allem bezüglich Atemwegssystem, Magen-Darm-Trakt und Mundgesundheit). Neben den klinisch manifesten Erkrankungen steht die Untersuchung subklinischer Vorstufen (zum Beispiel durch Messung der kognitiven Funktion, der Lungenfunktion oder der Intima-Media-Dicke der Halsschlagader) und funktioneller Veränderungen (zum Beispiel muskuloskelettale Untersuchungen) im Fokus. Zu den Risikofaktoren bzw. Expositionen, die besondere Beachtung finden werden, zählen Lebensstilfaktoren (zum Beispiel körperliche Aktivität und Fitness, Ernährung), psychosoziale und sozioökonomische Faktoren sowie genetische Faktoren und Umweltbedingungen [13]. Beispiele für einzelne wissenschaftliche Fragestellungen, die sich mithilfe der Nationalen Kohorte beantworten lassen werden, finden sich im wissenschaftlichen Konzept der Nationalen Kohorte [13].

\section{Ausgangsbevölkerung und Studienregion}

Die Studienbevölkerung für die Nationale Kohorte wird in insgesamt 18 Studienzentren über ganz Deutschland verteilt rekrutiert (- Abb. 1). Aus den regionalen Einwohnermelderegistern wird eine nach Alter und Geschlecht stratifizierte Zufallsstichprobe der Allgemeinbevölkerung im Alter von 20 bis 69 Jahren gezogen, um insgesamt 200.000 Personen, davon 100.000 Frauen und 100.000 Männer, $\mathrm{zu}$ untersuchen [13]. Es wird ein Beteiligungsanteil von $50 \%$ angestrebt, das heißt, die Einwohnermelderegisterstichprobe umfasst ca. 400.000 Personen (Bruttostichprobe). Die Ziehung dieser Stichprobe findet voraussichtlich zu zwei Zeitpunkten statt, zu Beginn und nach 2,5 Jahren.

Es erfolgt eine Anreicherung höherer Altersgruppen. So sind die beiden Dekaden 20-29 Jahre und 30-39 Jahre mit je $10 \%$ in der Kohorte vertreten, die älteren Dekaden mit einem Anteil von je 26,7\%. Dieser höhere Anteil an 40- bis 69-Jährigen ist durch das größere Risiko gerechtfertigt, in diesem Alter chronische Krankheiten in der näheren Zukunft zu entwickeln. Um die Untersuchung früher Krankheitsstadien (intermediäre Phäno- 
typen, frühe Funktionsstörungen, subklinische Krankheitsstadien) zu ermöglichen, werden aber auch Personen ab 20 Jahren eingeschlossen. Diese Verteilung nach Alter und Geschlecht wird in jedem Studienzentrum gleich sein.

Eine Unterstichprobe von 40.000 Personen wird ein zusätzliches beziehungsweise umfangreicheres Untersuchungsprogramm durchlaufen. Diese Personen werden nach dem Zufallsprinzip ausgewählt, jedoch entsprechend der prozentualen Alters- und Geschlechtsverteilung der Gesamtstichprobe. Die zufällige $\mathrm{Zu}$ ordnung zu dieser Gruppe erfolgt vor der Kontaktaufnahme, da sich das Informationsmaterial für diese von dem für die Basisgruppe unterscheidet.

Der gewählte Studienumfang stellt einen Kompromiss dar, der auf Schätzungen der Zahl der Neuerkrankungen an wichtigen Krankheitsbildern und der Häufigkeit und Verteilung wichtiger Risikofaktoren basiert. Zusätzlich sind natürlich auch finanzielle Aspekte eingeflossen.

Umfangreiche, komplexe Fallzahlabschätzungen sind im Anhang des Antrags dargestellt [13]. Beispielhaft werden nach zehn Jahren Follow-up folgende Zahlen an Neuerkrankungen erwartet: für Herzinfarkt ca. 5000, für Diabetes ca. 9000 , für Brustkrebs ca. 3600, für Lungenkrebs ca. 1100.

\section{Rekrutierung der \\ Studienteilnehmer}

Die Personen aus den Bevölkerungsstichproben werden in einem mehrstufigen Einladungsverfahren in die Studienzentren eingeladen. Als Erstes wird eine schriftliche Einladung auf dem Postweg verschickt. Dieser Brief enthält ein Einladungsanschreiben, eine Beschreibung der Ziele und Untersuchungselemente der Studie, den Hinweis auf die $\mathrm{Zu}$ stimmung des Datenschutzbeauftragten und der Ethikkommission und möglicherweise Unterstützungsbriefe regionaler Behörden und der Schirmherren des Projekts. Personen, deren Telefonnummer aus öffentlichen Telefonbüchern bekannt ist, werden im Verlauf der folgenden Tage vom Studienpersonal angerufen. Gleichzeitig wird den Personen die Telefonnummer des lokalen Studienzen-

\section{H.-E. Wichmann · R. Kaaks · W. Hoffmann · K.-H. Jöckel · K.H. Greiser · J. Linseisen Die Nationale Kohorte}

\begin{abstract}
Zusammenfassung
Die Nationale Kohorte (NK) ist ein gemeinsames interdisziplinäres Vorhaben von Wissenschaftlern aus der Helmholtz-Gemeinschaft, den Universitäten und anderen Forschungsinstituten in Deutschland. Ihr Ziel ist es, die Entstehung der wichtigsten chronischen Krankheiten (Krankheiten des HerzKreislauf-Systems und der Lunge, Diabetes, Krebs, neurodegenerative/-psychiatrische und Infektionskrankheiten), ihre subklinischen Vorstufen und auftretende funktionelle Veränderungen zu untersuchen. Die NK soll 200.000 Männer und Frauen im Alter von 2069 Jahren umfassen, die in 18 Studienzentren aus einer repräsentativen Stichprobe der Bevölkerung ausgewählt werden. Zusätzlich zu Interviews und Fragebogenerhebungen werden eine Reihe medizinischer Untersuchungen durchgeführt und verschiedene Bioproben gesammelt. Bei $20 \%$ der Personen wird
\end{abstract}

\section{The German National Cohort}

\section{Abstract}

The German National Cohort (GNC) is a joint interdisciplinary endeavour of scientists from the Helmholtz Association, universities and other German research institutes. Its aim is to investigate the development of major chronic diseases (cardiovascular diseases, cancer, diabetes, neurodegenerative psychiatric diseases, pulmonary and infectious diseases), the subclinical stages and functional changes. In 18 study centres across Germany, a representative sample of the general population will be drawn to recruit in total 200,000 men and women aged 20-69 years. In addition to interviews and questionnaires, the baseline assessment includes a series of medical examinations and the collection of a diverse range of biomaterials. In $20 \%$ of the participants, an intensified assessment programme

trums genannt, um Termine zu vereinbaren oder um zusätzliche Informationen erfragen zu können. Wenn innerhalb von vier Wochen kein Kontakt hergestellt werden konnte, wird eine Erinnerungseinladung per Post geschickt zusammen mit eine $\backslash \mathrm{r}$ vorgefertigten, frankierten Antwortkarte und mit Terminvorschlägen für den Besuch des Studienzentrums. Sowohl bei Telefonanrufen als auch Hausbesuchen wird dem Anteil der arbeitenden Bevölke- ein noch ausführlicheres Programm eingesetzt. Ferner werden etwa 40.000 Probanden eine Untersuchung mittels Magnetresonanztomographie (MRT) erhalten. Nach fünf Jahren werden alle Teilnehmer nochmals untersucht. Postalische Nachbefragungen zur Gesundheitssituation werden alle zwei bis drei Jahre erfolgen. Mit der NK wird eine herausragende Basis für die zukünftige epidemiologische Forschung in Deutschland geschaffen. Ihre Ergebnisse werden neue Möglichkeiten zur Prävention, Vorhersage und Früherkennung der wichtigsten Volkskrankheiten eröffnen.

\section{Schlüsselwörter}

Bevölkerungsbezogene Kohorte .

Chronische Krankheiten - Lebensstilfaktoren . Magnetresonanztomographie · Subklinische Vorstufen · Funktionelle Veränderungen

is foreseen. Also in 40,000 participants, magnetic resonance imaging of the whole body, heart and brain will be performed. After 5 years, a follow-up examination will be performed in all subjects and active follow-up by postal questionnaires is planned every $2-3$ years. The GNC will provide an excellent basis for future population-based epidemiology in Germany and results will help identify new and tailored strategies for prevention, prediction and early detection of major diseases.

\section{Keywords}

Population-based cohort . Chronic disease . Life-style factors · Magnet resonance imaging · Subclinical stages - Functional changes

rung Rechnung getragen, da sie nicht ausschließlich tagsüber stattfinden, sondern auch abends und am Wochenende.

Um eine hohe Teilnahme zu erreichen, werden verschiedene Maßnahmen ergriffen. Es wird Öffentlichkeitsarbeit betrieben werden, um den Bekanntheitsgrad der Nationalen Kohorte in der Bevölkerung zu erhöhen. Dies beinhaltet auch die Informationsweitergabe an und die Integration regionaler Allgemeinärzte und Apothe- 
Tab. 1 Nationale Kohorte: Studiendesign und Zeitplan

\begin{tabular}{|l|}
\hline Studiendesign \\
\hline - Bevölkerungsbezogene prospektive Kohorte (Männer und Frauen) \\
\hline - Alter 20 bis 69 Jahre \\
\hline - Zufallsstichprobe von Einwohnern definierter geografischer Regionen \\
\hline - 18 Studienzentren \\
\hline - Level 1, $n=200.000$ \\
\hline - Level 2, $n=40.000$ \\
\hline - Level $3, n=$ variabel (Zusätzliche Forschungsfragen mit zusätzlicher Finanzierung) \\
\hline - Magnetresonanztomographie (MRT)-Programm, $n=40.000$ \\
\hline - Basisuntersuchung mit Interviews, Fragebogen-Modulen, körperlicher Untersuchung, Tests der \\
kognitiven Funktion, Sammlung von Bioproben (Blut, Urin, Speichel, Nasenabstriche, Stuhl) \\
\hline 2,5 Stunden Untersuchungsprogramm für Level 1 und \\
4 Stunden intensiviertes Untersuchungsprogramm für Level 2 \\
\hline - Nach 5 Jahren Folgeuntersuchung bei allen Studienteilnehmern \\
\hline - Kalibrierungs-Substudie (Messungswiederholungen sowohl innerhalb der Basisuntersuchung als \\
auch der Folgeuntersuchung) \\
\hline - Kombination von aktiver (postalische Fragebögen alle 2-3 Jahre) und passiver Nachbeobachtung \\
(Verknüpfung mit Registern) \\
\hline Zeitplan \\
\hline - 2009-2012 Planung, Pretests \\
\hline - 2013 Pilotstudie \\
\hline$-2013-2017$ Basisuntersuchung \\
\hline - 2018-2022 Folgeuntersuchung \\
\hline - Ab 2015 Aktive Nachbeobachtung, alle 2-3 Jahre (Fragebögen per Post) \\
\hline - Ab 2018 Nutzung für epidemiologische Studien \\
\hline
\end{tabular}

Tab. 2 Nationale Kohorte: Fragebögen

\begin{tabular}{|ll}
\hline Allgemeine Fragebögen & Spezielle Fragebögen \\
\hline $\begin{array}{l}\text { - Soziodemografische und sozioökonomische } \\
\text { Faktoren }\end{array}$ & $\begin{array}{l}\text {-Neurologische und psychiatrische Faktoren } \\
\text { (Symptomfragebögen zu Depression, Angststö- } \\
\text { rung, Kopfschmerz und Schlaf) }\end{array}$ \\
\hline - Medizinische Anamnese & $\begin{array}{l}\text {-Psychosoziale Faktoren (Persönlichkeit, chroni- } \\
\text { scher Stress, Trauma, beruflicher Stress, soziales } \\
\text { Netzwerk; Konflikte im Beruf und in der Familie, } \\
\text { berufliche Unsicherheit) }\end{array}$ \\
\hline - Familienanamnese & - Infektionen und Immunfunktion \\
\hline - Medikation der letzten sieben Tage & -Schmerzen im Muskel-Skelett-System \\
\hline -Teilnahme an Screening-Untersuchungen & - Mundgesundheit \\
\hline - Frauen-/Männergesundheit & - Körperliche Aktivität (Beruf, Freizeit, Sport) \\
\hline - Rauchen, Alkohol, weitere Drogen & - Ernährung \\
\hline - Gesundheitsbezogene Lebensqualität & - Umweltfaktoren (Wohnungsadresse, Berufs- \\
& adresse) \\
\hline - Weitere persönliche Charakteristika & - Beruf (Screening spezieller Arbeitsbedingun- \\
& gen) \\
\hline & - Nutzung des Gesundheitssystems (Arzt- \\
& besuche, Krankenhausaufenthalte) \\
\hline aDie Machbarkeit wird derzeit zum Teil noch erprobt. & \\
\hline
\end{tabular}

ken sowie die Einrichtung eines Servicetelefons und einer Homepage im Internet. Zudem wird es Informationskampagnen in Printmedien, Radio und Fernsehen geben. Wichtige Anreize für die individuelle Teilnahme stellt beispielsweise die Mitteilung der eigenen Ergebnisse der befragt, medizinisch untersucht und um die Abgabe von Biomaterialien gebeten. Die Befragung erfolgt über ein computergestütztes persönliches Interview sowie mittels Fragebögen, die zum Teil im Untersuchungszentrum am TouchscreenComputer ausgefüllt werden und zum Teil von zu Hause im Internet oder als Papierversion ausgefüllt werden können. Eine Folgeuntersuchung (Nachuntersuchung) aller Probanden ist nach fünf Jahren geplant, ergänzt durch postalische Befragungen (Nachbeobachtung) alle zwei bis drei Jahre. Die Durchführung der Basisund Folgeuntersuchung wird insgesamt zehn Jahre umfassen (• Tab. 1). Die im Laufe der Nachbeobachtung über weitere zehn bis 20 Jahre bei einigen Teilnehmern auftretenden Erkrankungen können dann mit den zuvor zur Basisuntersuchung bzw. zur Folgeuntersuchung erhobenen Daten in Verbindung gebracht werden.

\section{Basisuntersuchung}

In der Intensitätsstufe 1 (Level 1), die von allen 200.000 Teilnehmern durchlaufen wird und ca. 2,5 Stunden dauert, werden Interviews und Fragebögen ( $\bullet$ Tab. 2) sowie verschiedene Untersuchungsverfahren (- Tab. 3) eingesetzt. In der Befragung werden Informationen $\mathrm{zu}$ soziodemografischen Faktoren, zu Lebensgewohnheiten (zum Beispiel körperliche Aktivität, Rauchen, Ernährung, Beruf), zu psychosozialen und sozioökonomischen Faktoren, zur medizinischen Anamnese und zur Familienanamnese erhoben.

Die Untersuchungen umfassen Messungen anthropometrischer Indizes, des Blutdrucks, der arteriellen Steifigkeit, ein Elektrokardiogramm (EKG), eine Spirometrie, Messungen der körperlichen Aktivität (Akzelerometrie) und Fitness, der kognitiven Funktionen und die Erfassung des Zahnstatus. Ein weiteres wichtiges Element ist die Sammlung von Biomaterialien von allen Teilnehmern. Die Protokolle zur Probenentnahme und Lagerung von Aliquots werden so geplant, dass sie die größtmögliche Auswahl für zukünftige Laboranalysen erlauben. Die Bioproben umfassen Blutproben, Plasma, Serum, Dimethylsulfoxid-präparierte lebende weiße Blutzellen (in einer Untergruppe), Erythrozyten, Urin, Speichel, Nasen- 
Tab. 3 Nationale Kohorte: Untersuchungsmodule und Bioproben

\begin{tabular}{|c|c|}
\hline Untersuchungsmodule ${ }^{a}$ & Bioproben $^{a}$ \\
\hline $\begin{array}{l}\text { - Herz-Kreislauf-System [Blutdruck, Herzfre- } \\
\text { quenz, EKG (10 s, } 12 \text { Ableitungen; } 24 \text { h), Carotis- } \\
\text { Sonographie (Intima-Media-Dicke)] }\end{array}$ & $\begin{array}{l}\text { - Biomaterialien [Serum, Blut, Plasma, Erythro- } \\
\text { zyten, Desoxyribonukleinsäure (DNA), Ribonuk- } \\
\text { leinsäure (RNA), lebende Zellen, Urin, Speichel, } \\
\text { Nasenabstrich, Stuhl] }\end{array}$ \\
\hline $\begin{array}{l}\text { - Diabetes [Oraler Glukose-Toleranz-Test (OGTT), } \\
\text { Advanced-Glycation-Endproducts (AGE)-Mes- } \\
\text { sung der Haut] }\end{array}$ & $\begin{array}{l}\text { - Umfang: } 20 \text { Mio. Aliquots, } 100 \text { Aliquots pro } \\
\text { Proband }\end{array}$ \\
\hline - Kognitive Funktion (Testbatterie) & $\begin{array}{l}\text { - Zentrales Biorepository (insgesamt } 2 / 3 \text { aller } \\
\text { Proben, dabei } 1 / 6 \text { im automatischen Lager, } 1 / 2 \\
\text { im zentralen Flüssigstickstoff-Lager) }\end{array}$ \\
\hline $\begin{array}{l}\text { - Lungenfunktion (Spirometrie, exhaliertes } \\
\text { Stickstoffmonoxid) }\end{array}$ & $\begin{array}{l}\text { - Regionale Flüssigstickstoff-Lagerung ( } 1 / 3 \text { aller } \\
\text { Proben) }\end{array}$ \\
\hline $\begin{array}{l}\text { - Muskel-Skelett-System (medizinische Unter- } \\
\text { suchung) }\end{array}$ & Magnetresonanztomographie (MRT) ${ }^{\mathrm{a}}$ \\
\hline $\begin{array}{l}\text { - Mundgesundheit (Zahnstatus, Untersuchung } \\
\text { der Mundhöhle) }\end{array}$ & -Ganzkörper-MRT \\
\hline $\begin{array}{l}\text { - Sinnesorgane (Augenuntersuchung, Hörtest, } \\
\text { Riechtest) }\end{array}$ & - Herz-MRT \\
\hline $\begin{array}{l}\text { - Körperliche Aktivität und Fitness (7-Tage-Ak- } \\
\text { zelerometrie, kardiorespiratorischer Fitness-Test, } \\
\text { Handgreifstärke) }\end{array}$ & -Gehirn-MRT \\
\hline $\begin{array}{l}\text { - Anthropometrie (Körpergewicht, Körpergröße, } \\
\text { Taillen- und Hüftumfang) }\end{array}$ & \\
\hline aDie Machbarkeit wird derzeit zum Teil noch erprobt. & \\
\hline
\end{tabular}

abstriche und Stuhlproben, die in einem zentralen Biorepository mit dezentraler Backup-Lagerung langfristig gelagert wer$\operatorname{den}(\cdot$ Tab. 3).

In der Intensitätsstufe 2 (Level 2) wird bei einer repräsentativen Untergruppe der Kohorte (20\%, 40.000 Teilnehmer) ein umfangreicheres Untersuchungsprogramm von insgesamt vier Stunden durchgeführt (• Tab. 1). Zusätzlich zu den Untersuchungen aus Level 1 sollen eine Carotis-Sonographie mit Messung der Intima-Media-Dicke, ein LangzeitEKG und erweiterte motorische, sensorische und kognitive Funktionstests durchgeführt werden. Außerdem wird ein Marker für Atemwegsentzündung gemessen, es werden die Mundhöhle und der Bewegungsapparat untersucht und zusätzliche Fragebögen eingesetzt.

Ein weiterer Programmpunkt ist die Magnetresonanztomographie (MRT). Diese wird an ca. 40.000 Personen durchgeführt und umfasst eine Ganzkörperuntersuchung mit zusätzlichen speziellen Untersuchungselementen für Gehirn und Herz. Die MRT-Untersuchungen werden umfassende Daten liefern, die es gestatten, die Prävalenz und Inzidenz MRT-basierter Befunde und ihre räumliche Vertei-
Der Zeitraum, in dem die Folgeuntersuchungen durchgeführt werden, beträgt - analog zur Basisuntersuchung - fünf Jahre, sodass im Mittel jede Person fünf Jahre nach Eintritt in die Studie ein zweites Mal untersucht werden kann. Auf Basis der Erfahrungen aus anderen deutschen Kohortenstudien, wird bei der Folgeuntersuchung von einer Beteiligung von $80 \%$ ausgegangen $[4,5]$.

In der Folgeuntersuchung wird ein großer Teil der Untersuchungen aus der Basisuntersuchung erneut durchgeführt. In dieser werden Veränderungen bei den Risikofaktoren, bei quantitativen Parametern der präklinischen Morbidität und bei anderen funktionellen Parametern sowie neu aufgetretene Krankheitsdiagnosen erfasst.

\section{Kalibrierungs-/ \\ Reliabilitäts-Substudie}

Zusätzlich zur mittelfristigen (Fünf-Jahres-)Wiederholung von Messungen im Rahmen der Folgeuntersuchung werden Kurzzeit-Wiederholungsmessungen innerhalb eines Jahres (im Mittel 6 Monate) nach der Erstuntersuchung durchgeführt. Sie werden an einer Subgruppe von 6000 Personen aus der Basisuntersuchung und von 4000 Personen aus der Folgeuntersuchung durchgeführt. Diese Kalibrierungs-/Reliabilitäts-Substudien dienen dazu, intraindividuelle Zufallsvariation bei den unterschiedlichen Messungen $\mathrm{zu}$ bestimmen, um bei den statistischen Auswertungen Korrekturen für Abschwächungseffekte zu ermöglichen, die sich aus der zufälligen Fehlklassifizierung ergeben.

\section{Methoden der Nachbeobachtung (Follow-up)}

Neben den beiden Untersuchungen (Basis- und Folgeuntersuchung) im Studienzentrum werden die Studienteilnehmer alle zwei bis drei Jahre kontaktiert und gebeten, Kurzfragebögen auszufüllen. Mit dieser werden Veränderungen im Lebensstil und anderer gesundheitsrelevanter Charakteristika (Medikamenteneinnahme, Rauchen, Menopause, ausgewählte Krankheitssymptome) und das Neuauftreten ausgewählter, wichtiger Erkran- 
kungen erfasst („aktives“ Follow-up), und es wird für wichtige Entitäten durch Einsichtnahme in medizinische Unterlagen validiert. Bei Krebserkrankungen wird ein Abgleich mit existierenden Krebsregistern durchgeführt („passives“ Follow-up). Parallel dazu wird alle zwei bis drei Jahre ein Mortalitäts-Follow-up mithilfe der Einwohnermelderegister durchgeführt.

Darüber hinaus wird eine Verknüpfung mit weiteren Sekundärdaten zur gesundheitlichen Situation und Versorgung angestrebt, zum Beispiel mit Krankenversicherungsdaten.

Für die interne Validität der Ergebnisse aus der Nationalen Kohorte ist eine möglichst geringe Ausfallrate (loss to follow-up) entscheidend. Aufgrund der sehr positiven Erfahrungen in bestehenden deutschen Kohortenstudien wird auch für die Nationale Kohorte von einer Beteiligungsrate an der Nachbeobachtung von über $80 \%$ ausgegangen. Von besonderer Bedeutung ist hierfür die kontinuierliche Kommunikation mit den Studienteilnehmern.

\section{Lagerung von Biomaterialien (Biobanking)}

Der größte Teil der Bioproben, die während der Basisuntersuchung und der Nachuntersuchung gesammelt werden, wird in einer zentralen Biobank (Biorepository) der Nationalen Kohorte eingelagert, die am Helmholtz-Zentrum München eingerichtet wird und ein vollautomatisches Lagersystem und ein manuelles Lager in Flüssigstickstoff-Tanks umfasst. Eine zusätzliche lokale Back-up-Lagerung (in den Clustern beziehungsweise Studienzentren) ist für ein Drittel der Proben geplant (- Tab. 3). Für alle inzidenten Fälle wichtiger Krebserkrankungen ist die systematische Sammlung von Tumorgewebe vorgesehen, das in einer zentralen Tumorbank am Deutschen Krebsforschungszentrum in Heidelberg gelagert werden wird.

\section{Ethik und Datenschutz}

Die Nationale Kohorte wird auf der Grundlage der deutschen Datenschutzgesetze [16] und weiterer Vorschriften durchgeführt. Ferner werden die Emp- fehlungen des Deutschen Ethikrats zu Forschungsbiobanken [17] sowie andere ethisch relevante Richtlinien zugrunde gelegt. Wichtiges Ziel ist die Sicherstellung des Vertrauens in die Art der Datensammlung und Speicherung. Das Konzept der Nationalen Kohorte wurde in enger Abstimmung mit dem „Arbeitskreis Wissenschaft" der Datenschutzbeauftragten auf Grundlage der Empfehlungen des Deutschen Ethikrats zu Biomaterialien entwickelt. Vor Beginn der Rekrutierung wird die finale Fassung des Studienprotokolls umfassend durch die jeweils verantwortlichen Datenschutzbeauftragten und Ethikkommissionen überprüft. Ein externer Ethikbeirat wird die Kohorte während ihrer gesamten Laufzeit begleiten.

\section{Qualitätssicherung und-kontrolle}

Um sicherzustellen, dass die Nationale Kohorte im Ganzen einschließlich Datenerhebung und Umgang mit den Daten sowie der Biomaterialgewinnung, -aufbereitung und -lagerung von standardisiert hoher Qualität ist - sowohl über die Zeit als auch über die 18 Studienzentren hinweg -, wird ein Qualitätsmanagementsystem etabliert werden. Eine Zertifizierung gemäß ISO 9001 wird angestrebt. Es wird sowohl ein internes als auch ein externes Qualitätsmanagement geben.

Für die Erhebung werden ausschließlich standardisierte Instrumente eingesetzt, und alle Untersuchungen werden anhand detailliert beschriebener Vorgehensweisen, das heißt anhand sogenannter Standard Operating Procedures (SOPs), durchgeführt. Das durchführende Personal wird umfangreich geschult und für jedes einzelne Element in der Erhebung zertifiziert. Über den Zeitverlauf werden in regelmäßigen Abständen wiederholte Trainings und die Erneuerung der Zertifizierung stattfinden. Ebenso erfolgt eine standardisierte Dokumentation der Abläufe im Studienzentrum. Unangekündigte Besuche zur Überprüfung der Abläufe und Untersuchungen im Studienzentrum werden durch ein internes Qualitätssicherungsteam durchgeführt werden. Die Qualitätskontrolle der Daten erfolgt auf lokaler und zentraler Ebene. Die beiden Integrationszentren sind für die zentrale Datenspeicherung und zugehörige Datenqualitätsüberprüfungen verantwortlich. Zusätzlich wird es sogenannte Kompetenzeinheiten zur Bearbeitung komplexer Studiendaten (zum Beispiel Akzelerometrie-Daten, EKG-Daten) geben, die auch für die jeweiligen qualitätssicherungsbezogenen Aspekte zuständig sind.

Für das externe Qualitätsmanagement wird eine unabhängige Expertengruppe beauftragt werden. Diese evaluiert engmaschig und systematisch die internen Qualitätssicherungsprozesse und leitet bei Auffälligkeiten die Informationen über notwendige Korrekturen rasch an die zuständigen Stellen weiter. Sowohl die interne als auch die externe Qualitätssicherung werden ihre Erkenntnisse in Berichten dokumentieren.

\section{Bisherige Ergebnisse und aktueller Stand}

Die Vorarbeiten zur Nationalen Kohorte laufen seit 2009. Ihr wissenschaftliches Konzept wurde im Februar 2011 beim Bundesministerium für Bildung und Forschung (BMBF) zur Begutachtung eingereicht. Die Begutachtung durch ein internationales Gremium erfolgte im April 2011. Alle wesentlichen Eckpunkte der Studie wurden von den Gutachtern sehr positiv bewertet.

Derzeit werden an den beteiligten Studienzentren Machbarkeitsstudien (Pretests) durchgeführt. Eine Gruppe von etwa 2000 Probanden, die über eine nach Alter und Geschlecht stratifizierte Einwohnermeldeamtsstichprobe ausgewählt wird, wird analog der geplanten Basisuntersuchung in den Studienzentren befragt und medizinisch untersucht; auch wird die Abnahme von Biomaterialien getestet. Ziel der Machbarkeitsstudien ist es, zentrale Funktionseinheiten (zum Beispiel Management der Bioproben, Datenmanagement und Qualitätskontrolle) einzurichten sowie Arbeitsabläufe zu entwickeln, zu testen und auf ihre Machbarkeit hin zu prüfen. Des Weiteren werden beispielsweise verschiedene Instrumente zur Datenerhebung und medizinische Untersuchungsgeräte auf ihre Verwendbarkeit und Praktikabilität in großen populationsbezogenen Kohortenstudien, ein- 
schließlich ihre Akzeptanz bei den Probanden, getestet, um die Auswahl der am besten geeigneten Instrumente zu ermöglichen und vorhandene Instrumente speziell für die Studienzwecke anzupassen.

Anhand der Empfehlungen der Gutachter und auf Basis der Ergebnisse der Machbarkeitsstudien ist das Konzept für die Nationale Kohorte bis zum Frühjahr 2012 überarbeitet worden und im Mai 2012 erneut begutachtet worden. Im Jahr 2013 ist die Durchführung der Pilotstudie geplant, die dann bereits das volle Untersuchungsprogramm für die Hauptstudie umfasst. Nach kleineren Korrekturen, die sich aus den Erfahrungen der Pilotstudie ableiten lassen, wird zügig mit der Hauptphase begonnen werden.

Ab 2013 sollen für die ersten zehn Jahre die benötigten Fördermittel zur Rekrutierung der Probanden gemeinsam vom BMBF, der Helmholtz-Gemeinschaft und den Ländern zur Verfügung gestellt werden.

Die Nutzung von Daten und Bioproben für epidemiologische Auswertungen kann beginnen, sobald die Basisuntersuchung (einschließlich Qualitätssicherung) abgeschlossen ist. Insgesamt soll die $\mathrm{Na}$ tionale Kohorte der Forschung für eine Periode von mindestens 25-30 Jahren zur Verfügung stehen.

\section{Fazit}

Die Nationale Kohorte wird wesentlich dazu beitragen, neue und zielgenaue Strategien zur Prävention, Vorhersage und Früherkennung von chronischen Krankheiten zu entwickeln. Ihr Beitrag wird durch vielfältige Synergien mit den deutschen Zentren für Gesundheitsforschung, die an vielen Standorten Patientenkohorten aufbauen, noch verstärkt werden. Die Nationale Kohorte stellt eine hervorragende Basis für die zukünftige epidemiologische Gesundheitsforschung in Deutschland dar und hebt sich im Vergleich zu großen internationalen Kohortenstudien in besonderer Weise ab, insbesondere hinsichtlich (i) der verstärkten Erfassung subklinischer Krankheitsmerkmale und funktioneller Messungen (in Ergänzung zu den klinischen Endpunkten), (ii) einer Nachuntersuchung aller 200.000 Studienteilnehmer, (iii) der Qua- lität und Diversität der gesammelten Biomaterialien und (iv) der Durchführung einer MRT-Untersuchung bei 40.000 Personen (und der damit einhergehenden Erstellung einer Datenbank zur Bildgebung).

Limitationen einer so breit angelegten Kohorte sind, dass (i) die Gesamtzahl komplexer und teurer Untersuchungsinstrumente aus Zeit- und Kostengründen eingeschränkt ist, (ii) sehr aufwändige Verfahren zum Erreichen einer hohen Beteiligung, nicht möglich sind und (iii) die Logistik und Qualitätssicherung durch die große Zahl von Studienzentren kompliziert wird.

Insgesamt überwiegen nach Ansicht der Autoren die Vorteile gegenüber den Limitationen deutlich. Die Nationale Kohorte stellt eine wichtige Investition in die Zukunft dar, wird von großem Nutzen für die medizinische Forschung sein und wichtige Erkenntnisse zur Verbesserung der Gesundheit der Bevölkerung liefern.

\section{Korrespondenzadresse}

Prof. Dr. Dr. H.-E. Wichmann

Helmholtz-Zentrum München Ingolstädter Landstr. 1, 85764 Neuherberg wichmann@helmholtz-muenchen.de

Danksagung. Wir danken den vielen Wissenschaftlerinnen und Wissenschaftlern, die durch ihre Beteiligung an den Thematischen Arbeitsgruppen maßgeblich an der Konzeptentwicklung der Nationalen Kohorte mitgewirkt haben. Für die Mitwirkung an dem vorliegenden Artikel danken wir Frau Dipl.-Geol. Inke Thiele, MPH. Des Weiteren sind wir den Mitarbeiterinnen und Mitarbeitern, die lokal und überregional am Aufbau der Infrastruktur und der Durchführung der Pretests beteiligt waren, zu großem Dank verpflichtet.

Interessenkonflikt. Der korrespondierende Autor gibt für sich und seine Koautoren an, dass kein Interessenkonflikt besteht.

\section{Literatur}

1. Robert Koch-Institut (Hrsg) (2006) Gesundheit in Deutschland. Gesundheitsberichterstattung des Bundes. Robert Koch-Institut, Berlin

2. Van Baal P, Polder J, Wit A de et al (2008) Lifetime medical costs of obesity: prevention no cure for increasing health expenditure. PLoS Med 5(2):29

3. Rappange DR, Brouwer WB, Rutten FF, Baal PH van (2010) Lifestyle intervention: from cost savings to value for money. J Public Health 32:440-447
4. Greiser KH, Kluttig A, Schumann B et al (2005) Cardiovascular disease, risk factors and heart rate variability in the elderly general population: design and objectives of the Cardiovascular disease Living and Ageing in Halle (CARLA) Study. BMC Cardiovasc Disord 5:33

5. Bergmann MM, Bussas U, Boeing H (2000) Follow-up procedures in EPIC Germany - data quality aspects. Ann Nutr Metab 43(4):225-234

6. Holle R, Happich M, Lowel H, Wichmann HE (2005) KORA - a research platform for population based health research. Gesundheitswesen 67 (Suppl 1):19-25

7. John U, Greiner B, Hensel E et al (2001) Study of Health In Pomerania (SHIP): a health examination survey in an east German region: objectives and design. Soz Praventivmed 46:186-194

8. Erbel R, Möhlenkamp S, Moebus S et al (2010) Coronary risk stratification, discrimination, and reclassification improvement based on quantification of subclinical coronary atherosclerosis: the Heinz Nixdorf Recall study. J Am Coll Cardiol 56:1397-1406

9. Nationale Kohorte (2012) Nationale Kohorte. http://www.nationale-kohorte.de/index.html

10. UK Biobank (2012) UK Biobank. http://www.ukbiobank.ac.uk/

11. Constances (2012) Constances. http://www.constances.fr/index.php?option $=$ com_rokdownloads \&view $=$ folder\&Itemid $=126 \&$ id $=237 \% 3$ Aannexes du-protocole-de-la-phase-pilote\&limitstart $=10$

12. LifeGene (2012) LifeGene. http://www.lifegene.se/ Forscientists/Questionnaire-Overview/

13. Nationale Kohorte (2012) Wissenschaftliches Konzept. http://www.nationale-kohorte.de/content/ wissenschaftliches_konzept_der_nationalen_kohorte.pdf

14. Nationale Kohorte (2012) EPC-Mitglieder. http:// www.nationale-kohorte.de/content/epc-mitglieder.pdf

15. Nationale Kohorte (2012) Thematische Arbeitsgruppen. http://www.nationale-kohorte.de/content/thematisch-ags.pdf

16. Bundesdatenschutzgesetz (2010) Bundesdatenschutzgesetz in der Fassung der Bekanntmachung vom 14. Januar 2003 (BGBI. I S. 66), das zuletzt durch Artikel 1 des Gesetzes vom 14. August 2009 (BGBI. IS. 2814) geändert worden ist. http://www. gesetze-iminternet.de/bundesrecht/bdsg_1990/ gesamt.pdf

17. Deutscher Ethikrat (2010) Humanbiobanken für die Forschung. Stellungnahme. http://www.ethikrat.org/dateien/pdf/stellungnahme-humanbiobanken-fuer-die-forschung.pdf 